\title{
Aplicación del programa "Galicia de reeducación de agresores de género" en el Estado de México
}

\author{
Implementation of the "Galician for the Re-education of Gender \\ Aggressors" program in Estado de México
}

\begin{abstract}
Aplicação do programa "Galiza para a reeducação dos agressores de gênero" no Estado do México
\end{abstract}

Elvira Ivone González Jaimes

Universidad Autónoma del Estado de México, México

ivonegj@hotmail.com

https://orcid.org/0000-0002-5328-5586

\section{Resumen}

El presente trabajo tiene como objetivo dar cuenta del impacto que tuvo el programa "Galicia de reeducación de agresores de género" en 65 participantes del género masculino tipificados como agresores primarios de violencia de género y atendidos en los Centros de Atención y Retención ubicados en los municipios de Atizapán, Ecatepec y Tlalnepantla del Estado de México. Se trata de un procedimiento psicológico enfocado en disminuir la agresión ante el género femenino y con ello contribuir a reducir la tasa de feminicidios. La intervención consistió en realizar una investigación documental de los casos para poder proceder a la aplicación del tratamiento adecuado (flexible y adaptable). La duración fue de entre seis a ocho meses, de acuerdo con las sanciones legales que estipulaban los casos. En los resultados se observó una disminución de la conducta agresiva en los participantes mediante la mejora de habilidades de solución de problemas cotidianos, de comunicación con el género femenino y la reducción de conductas violentas en contra el género femenino.

Palabras clave: feminicidio, programa de intervención, violencia de género, violencia familiar. 


\section{Abstract}

The present work aims to give an account of the impact that the "Galicia for the reeducation of gender aggressors" program had on 65 male participants classified as primary aggressors of gender violence and cared for in the Attention and Retention Centers located in the municipalities of Atizapán, Ecatepec and Tlalnepantla of the Estado de México. It is a psychological procedure focused on reducing aggression towards the female gender and thereby helping to reduce the rate of femicides. The intervention consisted of conducting a documentary investigation of the cases in order to proceed with the application of the appropriate treatment (flexible and adaptable). The duration was between six to eight months, in accordance with the legal sanctions stipulated in the cases. In the results, a decrease in aggressive behavior was observed in the participants through the improvement of everyday problem solving skills, communication with the female gender and the reduction of violent behaviors against the female gender.

Keywords: femicide, intervention program, gender-based violence, family violence.

\section{Resumo}

O presente trabalho pretende dar conta do impacto que o programa "Galiza para a reeducação dos agressores de gênero" teve em 65 participantes do sexo masculino classificados como principais agressores da violência de gênero e atendidos nos Centros de Atenção e Retenção localizados nos municípios de Atizapán., Ecatepec e Tlalnepantla do Estado do México. Trata-se de um procedimento psicológico voltado para a redução das agressões ao gênero feminino e, assim, contribuir para a redução do índice de feminicídios. A intervenção consistiu na realização de uma investigação documental dos casos de forma a proceder à aplicação do tratamento adequado (flexível e adaptável). A duração foi de seis a oito meses, de acordo com as sanções legais estipuladas nos processos. Nos resultados, observou-se uma diminuição do comportamento agressivo dos participantes por meio do aprimoramento das habilidades de resolução de problemas cotidianos, da comunicação com o gênero feminino e da redução de comportamentos violentos contra o gênero feminino.

Palavras-chave: feminicídio, programa de intervenção, violência de gênero, violência familiar.

Fecha Recepción: Junio 2020

Fecha Aceptación: Diciembre 2020 


\section{Introducción}

El presente trabajo es resultado de una investigación documental y de campo enfocada en tratar psicoterapéuticamente al agresor primario de violencia de género en México. Desafortunadamente, este tipo de violencia ocupa el primer lugar de agresiones contra las mujeres. De acuerdo con la Encuesta Nacional sobre la Dinámica de las Relaciones en los Hogares (Endireh) (citada en el Instituto Nacional de Estadística y Geografía [Inegi], 22 de noviembre de 2018), 19.1 millones de mujeres mayores de 15 años han enfrentado violencia por parte de su pareja, esposo o novio actual o último, a lo largo de su relación; en $64.0 \%$ de los casos se trata de violencia severa y muy severa. Al ser estadísticamente la pareja el agresor primario, con quien conviven rutinariamente, las mujeres se encuentran en un grado de vulnerabilidad preocupante. "Esta situación se ha mantenido en niveles similares durante los últimos 10 años” (Inegi, 22 de noviembre de 2018, p. 2). Aún más, la pareja ocupa el segundo lugar como causante de feminicidio, siguiendo de nueva cuenta los datos obtenidos por la Endireh (Inegi, 18 de agosto de 2017). En los primeros seis meses de 2020, de enero a junio, se registraron 1844 homicidios de mujeres, lo que confirma el dato de que en el país cada día son asesinadas 10 mujeres (García, 13 de febrero de 2021). En suma, aquí se tratará a la violencia de género por parte de la pareja como núcleo principal del feminicidio.

Más precisamente, esta investigación se realiza en el Estado de México, uno de los estados con mayor incidencia delictiva; tan solo el mes de marzo de 2021, de los 183111 presuntos delitos registrados, 34834 casos fueron reportados en el Estado de México (Gobierno de México, 2021).

La sociedad mexicana se distingue por un muy alto nivel de violencia de género inscrita en la vida cotidiana. La forma extrema de violencia es el feminicidio. La otrora Comisión de Derechos Humanos del Distrito Federal (ahora de la Ciudad de México) (2011), ponía en claro en una de las editoriales de la revista $d$ fensor lo siguiente:

La violencia feminicida en México obedece no solo a un contexto de cultura machista y misógina arraigada, sino también a una serie de factores sociales, económicos y políticos (discriminación por género, impunidad, condición social, edad, etnia y criminalidad, entre otros) que sistemáticamente vulneran todos los derechos de las mujeres al extremo de poner en peligro su integridad y causar su muerte (p. 3). 


\section{Revista Iberoamericana de las Ciencias Sociales y Humanísticas}

ISSN: $2395-7972$

Ahora bien, la alternativa de tratamiento preventivo y curativo que se propone en esta investigación se fundamenta en la última reforma del 13 abril del 2018 a la Ley General de Acceso de las Mujeres a una Vida Libre de Violencia, que a pie de letra dice:

Artículo 14. Las entidades federativas y el Distrito Federal, en función de sus atribuciones, tomarán en consideración:

(...) IV. Diseñar programas que brinden servicios reeducativos integrales para víctimas y agresores.

(...) Artículo 41. Son facultades y obligaciones de la Federación:

(...) VIII. Coordinar la creación de programas de reeducación y reinserción social con perspectiva de género para agresores de mujeres.

(...) Artículo 49. Corresponde a las entidades federativas y al Distrito Federal, de conformidad con lo dispuesto por esta ley y los ordenamientos locales aplicables en la materia:

(...) XII. Impulsar programas reeducativos integrales de los agresores (Congreso de la Unión, 14 de junio de 2012) (cursivas añadidas).

La directora del Instituto Nacional de las Mujeres (Inmujeres), Nadine Gasman, durante su presentación ante los medios en marzo del 2019, habló sobre la problemática y la inminente necesidad de erradicar el problema. Y para ello propuso: 1) protección a las mujeres del maltrato y 2) cambiar la forma de pensar del mexicano a través de programas para trabajar con la masculinidad.

Sin duda, ahora es el momento de dar alternativas de tratamiento probadas científicamente y ejecutadas con éxito por varios años en otras sociedades semejantes a la mexicana. En ese sentido, aquí se propone el programa "Galicia de reeducación de agresores de género".

\section{Violencia de género y el feminicidio}

Salvatierra (2007) menciona que el feminicidio es tan antiguo como el patriarcado. Este acto es ejecutado por hombres para asegurar el dominio masculino.

Por su parte, Romero (2014) rastrea e identifica que el tema emergió a raíz de los procesos judiciales emprendidos en contra de los perpetradores de crímenes y abusos durante la segunda guerra chino-japonesa. Durante la ocupación japonesa en el sur de China (1937), miles de civiles fueron masacrados, en su mayoría mujeres vejadas y asesinadas con lujo de 


\section{Revista Iberoamericana de las Ciencias Sociales y Humanísticas}

ISSN: $2395-7972$

crueldad. Y la discusión continuó con el evento de violaciones masivas de niñas y jovencitas ocurridas en Bangladés (1971) y la matanza de jóvenes universitarias en Montreal. Tomando en cuenta lo anterior, "[Diana] Russell define los feminicidios como: 'los crímenes perpetrados contra las mujeres por el simple hecho de serlo"” (Romero, 2014, p. 42).

En México, las evidencias y el registro sobre el feminicidio se han realizado desde el año 1993. Primeramente, el foco estuvo en Ciudad Juárez, Chihuahua, donde se comenzaron a presentar actos de violencia recurrentes hacia las mujeres que culminaron en feminicidios y antes los cuales las autoridades hacían caso omisión debido a la falta de sensibilidad (Romero, 2014).

En el Estado de México, los datos de averiguaciones previas permiten afirmar que $51 \%$ de las víctimas de violencia de género se encontraban en un rango de edad que va de los 16 a los 40 años, por lo que se posiciona como el sector de mayor riesgo, la etapa social y sexualmente más activa (Arteaga y Valdés, 2010). La mayoría de estas mujeres fueron asesinadas por su pareja (55\%), ya sea formal o informal (casada, divorciada, separada o en unión libre). "Mientras que las mujeres reportadas simplemente como solteras abarcan $28 \%$ de las actas ministeriales revisadas" (Arteaga y Valdés, 2010, p. 16).

Guerra (2018), tras recabar sistemáticamente datos, reporta 1559 feminicidios solo en 2016. Mujeres “destazadas, calcinadas, violadas, asesinadas a balazos, abandonadas en canales, ríos, terrenos, carreteras, desaparecidas, despojadas, denigradas, olvidadas, descalificadas" (Guerra, 2018, p. 21)

Además, comenta que es un fenómeno no tomado en su nivel de importancia por las autoridades y la sociedad. En efecto:

Solo $18.79 \%$ de los feminicidios en el país fueron "investigados"; 1155 de estos no tienen ni responsables ni detenidos; 76 presuntos culpables se encuentran prófugos y 35 se suicidaron después de asesinar a sus parejas o exparejas sobre las mujeres asesinadas (Guerra, 2018, p. 22).

Por otro lado, tenemos los registros de las instituciones gubernamentales. La Endireh $(2003,2006,2011,2016)$ es una fuente valiosa sobre la violencia contra las mujeres, ya que recolecta datos de instituciones públicas y académicas, organismos internacionales y sociedad civil, además de que suma diferentes objetivos y perspectivas, lo que proporciona una visión panorámica del problema social y sus connotaciones. Uno de sus datos más duros es que $40.1 \%$ de las mujeres han sufrido alguna agresión emocional por parte de su pareja, 
Revista Iberoamericana

de las Ciencias Sociales y

Humanísticas

ISSN: 2395 - 7972

$20.9 \%$ un acto violento relacionado con la economía o el patrimonio, $17.9 \%$ agresión física y $6.5 \%$ sexual.

Consecuentemente, por su alta incidencia en México, el Comité para la Eliminación de la Discriminación contra la Mujer (Cedaw) de la Organización de las Naciones Unidas [ONU] (2007) ha pedido que se adopten medidas urgentes para prevenir las muertes violentas, los homicidios y las desapariciones forzadas de mujeres. Y ha posicionado a México entre los primeros cinco países de Latinoamérica que presentan más feminicidios.

Las instancias internacionales como el comité de la ONU han instado al Gobierno de México a que tipifique el feminicidio como delito en todos los estados mexicanos y a la implementación de políticas para mitigar la trata de mujeres y niñas para la explotación sexual y el trabajo forzado. Asimismo, la Organización Mundial de la Salud [OMS] y la Organización Panamericana de la Salud [OPS] (22 de noviembre de 2017) sugieren fortalecer la vigilancia y la detección del femicidio y de la violencia por parte de la pareja (cuadro de factores de riesgo/nivel familiar relacional) y garantizar la documentación de la relación entre la víctima y el perpetrador.

\section{La vulnerabilidad aceptada propiciada por el agresor primario}

Existen diferentes tipos de feminicidio, según las circunstancias y el modus operandi, aunque la premisa general de "por razones de género" en todos los casos se mantiene. Julia Monárrez (2010), además de construir una importante base de datos de los feminicidios en Ciudad Juárez, tomó en cuenta la causa o el motivo del victimario y la relación de parentesco para categorizar las diferentes expresiones de los feminicidios. Aquí se rescata particularmente el denominado feminicidio íntimo: el asesinato de una mujer cometido por un hombre con quien la víctima tenía o había tenido una relación o un vinculo íntimo: marido, exmarido, compañero, novio, exnovio o amante (Monárrez, 2010). Esta relación en algún momento fue aceptada de forma expresa o por sometimiento (López, 2010).

Los crímenes de feminicidios son, claramente, crímenes de poder cuyo modelo conductual presenta una doble función: por un lado, la retención o manutención, y por el otro, la reproducción del poder (Moscovici, 2002). Por lo que no se considera un hecho aislado y cerrado en sí mismo. Son construcciones sociales tradicionales a través de las cuales hombres y mujeres se relacionan y mediante las cuales se perpetúa el machismo. De esta forma, los 


\section{Revista Iberoamericana}

de las Ciencias Sociales y

Humanísticas

ISSN: $2395-7972$

feminicidios no son solo la expresión de una crisis (social, económica o de valores), sino una manifestación de la concepción de las mujeres como objetos (Jiménez, 2011).

\section{Tipificación del feminicidio y violencia de género en México}

El artículo 325 del Código Penal Federal mexicano dispone lo siguiente: "Comete el delito de feminicidio quien prive de la vida a una mujer por razones de género" (Secretaría de Gobernación, 1 de julio de 2020, p. 99). Además: “A quien cometa el delito de feminicidio se le impondrán de cuarenta a sesenta años de prisión y de quinientos a mil días de multa" (Secretaría de Gobernación, 1 de julio de 2020, p. 100).

Aunado a lo anterior, en el artículo 343 Bis:

Comete el delito de violencia familiar quien lleve a cabo actos o conductas de dominio, control o agresión física, psicológica, patrimonial o económica, a alguna persona con la que se encuentre o haya estado unida por vínculo matrimonial, de parentesco por consanguinidad, afinidad o civil, concubinato, o una relación de pareja dentro o fuera del domicilio familiar.

A quien cometa el delito de violencia familiar se le impondrá de seis meses a cuatro años de prisión y perderá el derecho de pensión alimenticia. Asimismo, se le sujetará a tratamiento psicológico especializado (Secretaría de Gobernación, 1 de julio de 2020, p. 102).

Por su parte, en el Código Penal del Estado de México, además de tipificar el feminicidio de igual forma que en el federal, agrega lo siguiente:

VIII. Como resultado de violencia de género, pudiendo ser el sujeto activo persona conocida o desconocida y sin ningún tipo de relación.

En los casos a que se refiere este artículo [281], la penalidad será de cuarenta a setenta años de prisión o prisión vitalicia y de setecientos a cinco mil días de multa.

Además de las sanciones descritas en el presente artículo, el sujeto activo perderá todos los derechos con relación a la víctima, incluidos los de carácter sucesorio.

La pena se agravará hasta en un tercio cuando la víctima sea mujer menor de edad, embarazada o discapacitada, así como cuando el sujeto activo 


\section{Revista Iberoamericana}

de las Ciencias Sociales y

Humanísticas

ISSN: 2395 - 7972

sea servidor público y haya cometido la conducta valiéndose de esta condición (LIII Legislatura del Estado de México, 20 de marzo de 2020, p. 94).

Aunque en México se han dado grandes pasos con respecto a la adopción de estándares internacionales y leyes específicas de protección a los derechos de las mujeres en la legislación interna, por ejemplo, la creación del programa “Alerta de violencia de género contra las mujeres", un mecanismo de protección de los derechos humanos apoyado en Ley General de Acceso de las Mujeres a una Vida Libre de Violencia, sigue siendo insuficiente dado los altos índices de violencia en contra de ellas.

\section{Atención a agresores y a víctimas, respuesta a los artículos 325 y 343 Bis}

En México existen programas de intervención para las víctimas y también para los agresores dentro de las cárceles por lapsos intermitentes, dentro de pena privativa de libertad, otorgado por los servidores del sistema penitenciario federal con el objetivo de reeducar y lograr la reinserción social por rehabilitación. Los programas son extraídos de modelos europeos, pero han tenido poca difusión, además de que el registro sistematizado de sus beneficios no ha sido documentado científicamente. Por ejemplo, hubo un esfuerzo de utilizar el manual de Ruiz et al. (2010), creado en España, para desarrollar aspectos motivacionales y la perspectiva de género en un contexto penitenciario. También existe evidencia en expediente de casos del registro de programas de acceso voluntario y obligatorio para agresores de género desarrollados en la comunidad y realizados a la par de la protección a la víctima en casas de organizaciones sociales y el estado.

\section{Alternativa de tratamiento para agresores primarios}

La historia de los programas de asistencia para los agresores primarios de género empieza en la década de los 70 y estuvo en auge en la década de los 80. Los más reconocidos son el programa "Emerge” fundado en Boston, Massachusetts (1977); el "modelo de Duluth", implementado por primera vez en Minnesota (1981); el programa "Hombres contra la violencia de los hombres", iniciado en Hamburgo (1984); el programa "Respect", desarrollado en el Reino Unido, y el programa “Change”, implantado en Escocia (1989). Lo que se persigue en estas décadas es un cambio de paradigma social y jurídico propiciado principalmente por el movimiento feminista (Pérez, Giménez y Espinoza, 2012) 


\section{Revista Iberoamericana \\ de las Ciencias Sociales y Humanísticas}

ISSN: 2395 - 7972

Tomando en cuenta el artículo 343 Bis arriba citado, donde se estipula que al victimario "se le sujetará a tratamiento psicológico especializado" (Secretaría de Gobernación, 1 de julio de 2020, p. 102), se propone un programa, el “Galicia de reeducación de maltratadores de género", basado, probado y ejecutado principalmente en la región de Galicia, España, y emergido de trabajos como el de Ruiz et al. (2010), Lila, Gracia y Herrero (2012) y Arce y Fariña (2010). Las virtudes del programa es que trata características sociolegales específicas. Además, es posible adaptar el diseño del tratamiento al diagnóstico elaborado: revisión de expedientes judiciales o psicológicos conductuales, incluyendo entrevistas y baterías de pruebas psicométricas. El avance del tratamiento va ir de acuerdo con un monitoreo evaluativo conductual, asociado a las ideas de relación de pareja y sus circunstancias (Arce y Fariña, 2010).

\section{Tratamiento psicológico especializado utilizado}

Arce, Fariña, Vázquez, Novo y Seijo (2015) enfatizan en que las intervenciones de este tipo deben ser ajustables a las necesidades y evolución del caso. Si se ejecutan correctamente, pueden contribuir a la disminución de la frecuencia de conductas de maltrato y a la prevención de recaídas (Arce y Fariña, 2010).

La ficha técnica del programa es la siguiente:

1) Está diseñado para adultos agresores primarios de violencia doméstica con inteligencia y salud mental dentro de parámetros normales.

2) La intervención puede ser grupal, individual o mixta.

3) El contexto de intervención, medio abierto o cerrado.

4) Se consideran medidas para reincidencias (informes conductuales externos e informes de la víctima).

5) Los períodos de tratamiento dependen de la evolución del acusado, avalados por registros de sesiones.

6) Se recomienda un periodo de seguimiento (intermitente o continuo) de seis meses hasta varios años. 
Revista Iberoamericana

de las Ciencias Sociales y

Humanísticas

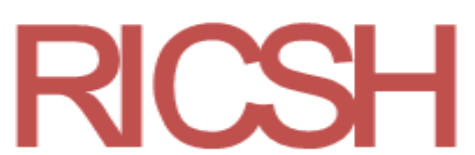

ISSN: 2395 - 7972

\section{Método}

Se trata de una investigación cuasiexperimental. La muestra estuvo conformada por 65 participantes del género masculino tipificados como agresores primarios de género, quienes asistieron a una intervención o tratamiento de siete módulos (especificados en procedimiento) y evaluaciones documentales y psicométricas por módulos. Los datos obtenidos fueron sometidos a un análisis cuantitativo utilizando estadística descriptiva.

\section{Pregunta de investigación}

- ¿Conocer el impacto que tiene el programa "Galicia de reeducación de maltratadores de género" en la disminución de comportamiento violento contra el género femenino?

\section{Participantes}

Se trabajó con un grupo de 65 participantes del género masculino asignado por las autoridades gubernamentales, con sentencia de asistir a pláticas o tratamiento psicológico especializado en centros de atención municipal. Sus características principales son: adultos jóvenes, entre 26 a 35 años, casados, con nivel académico básico y nivel económico bajo, la relación con su pareja es eventual por sentencia y provenientes de los municipios de Atizapán, Ecatepec y Tlalnepantla, del Estado de México.

\section{Procedimiento}

1) Investigación documental. Se revisaron los expedientes de los participantes localizados en los archivos de los Centros de Atención y Reeducación para Personas que Ejercen Violencia de Género. Y se observó que estos fueron elaborados a partir de los oficios de canalización de la instancia correspondiente, dirigidos al Consejo Estatal de la Mujer y Bienestar Social. Dentro de los expedientes se observa un reporte general expedido por la autoridad en turno de la violencia de género, la injusticia social o violación de derechos humanos. La autoridad del Centro de Atención es la que asigna la asistencia a pláticas, conferencias o talleres, así como el tiempo de participación, apegándose al caso (procedimientos legales, dimensión y gravedad del conflicto intrafamiliar) y haciendo hincapié en la violencia de género del agresor primario y el manejo intrainstitucional. 


\section{Revista Iberoamericana \\ de las Ciencias Sociales y Humanísticas}

ISSN: $2395-7972$

2) Intervención en campo. Se observaron las diferentes dinámicas que se llevaban con los agresores primarios de género en los centros de atención:

a) Seguimiento de las sentencias dictadas por las autoridades para generar las actividades específicas dependiendo del caso en tiempo y forma.

b) Designación a las actividades preestablecida que genera el Centro de Atención

c) Ajuste personalizado.

3) Las evaluaciones e intervención fueron apegadas a los lineamientos del Código ético del psicólogo (Sociedad Mexicana de Psicología, 2010). Desde el principio se les proporcionó a los participantes la hoja de consentimiento informado y responsabilidad para ser firmada.

4) Evaluación de la muestra para obtener el diagnóstico:

a) Entrevista semiestructurada para maltratadores de género (Arce y Fariña, 2005). Se obtuvo información sociodemográfica, apoyo social, descripción de episodios del maltrato y motivación para el cambio e interpretación personal de su situación actual.

b) Psicometría sistematizada (elaborada por ingenieros en Sistemas de la Universidad Autónoma del Estado de México, campus Zumpango)

I) Personalidad con el "Inventario multifásico de personalidad de Minnesota, R-2” (con siglas en inglés MMPI-R-2). Se aplicó todo el inventario o solo las escalas sugeridas por la entrevista de acuerdo con el caso (Butcher, et al., 1995).

II) La inteligencia y habilidad mental general se evaluó a través del "Test de matrices progresivas" de Raven (2015).

III) Adicciones a través de la "Prueba de detección de consumo de alcohol, tabaco y sustancias", elaborada por la OMS (2011), traducida y adaptada por la OPS.

5) El tratamiento fue ejecutado de forma individual, grupal o mixta, con seis módulos, en 30 sesiones (una sesión por semana) y tiempo total de tratamiento de seis a ocho meses.

6) Las estadísticas utilizadas fueron descriptivas para obtener las características de la muestra, así como la aplicación del tratamiento. Principalmente se utilizó la medida de porcentaje, porque sirve como unidad para comparar la población. 


\section{Aplicación del tratamiento por módulos}

1) Establecimiento del diagnóstico. Realización de entrevistas y evaluaciones psicométricas para generar líneas maestras de la programación que serán ajustadas de acuerdo con la evolución del agresor primario incluyendo: a) orientación terapéutica multimodal (cognitiva y comportamental), b) multinivel de acuerdo con el caso (individual, familiar, laboral y sociocomunitaria y $c$ ) obligaciones que ha de cumplir por ingresar al programa.

2) Responsabilización de las conductas violentas. Se incluye la gestión de la seguridad y control del riesgo, de carácter ordinario y extraordinario.

3) Concienciación sobre el ciclo de la violencia. Se muestra la relación entre situaciones, pensamientos, emociones y conductas a través de juego de roles para identificar las situaciones generadoras de tensión.

Al final del módulo se realiza una reevaluación psicológica, conductual y familiar para ajustar y orientar el tratamiento

4) Perspectiva de género. Se realiza la confrontación y reestructuración de creencias erróneas acerca del género. $\mathrm{Y}$ un automonitoreo que sirve para observar: progreso, adherencia al tratamiento y conductas simuladas.

5) Competencia personal y social. Se hace una evaluación y autoevaluación de sus capacidades personales, sociales y sus relaciones interpersonales. Aquí se realizó el monitoreo de corte. Asimismo, se ubicaron las unidades de tratamiento "Resolución de problemas" y "Habilidades de comunicación" por ser puntos clave para disminuir la violencia de género en el agresor primario.

6) Concienciación sobre el ciclo de la violencia. La responsabilidad en el comportamiento violento para el afrontamiento y prevención de recaídas fue asignada. Cuando el sujeto puede resolver problemas de la vida cotidiana y comunicar sus pensamientos y emociones entonces se hace responsable de su conducta.

7) Cierre y programación de seguimiento. Reevaluación de todas las esferas y retroalimentación verbal y escrita interinstitucional para el seguimiento del caso.

Como se puede observar, es un programa completo que trata diferentes aristas del agresor primario de género, el cual fue aplicado por primera vez en 2005 y fue perfeccionándose hasta el 2015, cuando fue publicado. Este programa asume la perspectiva 
Revista Iberoamericana

de las Ciencias Sociales y

Humanísticas

ISSN: $2395-7972$

de la justicia terapéutica: establece un nexo entre el proceso judicial y la reeducación del penado (Arce, Fariña y Novo, 2014).

Las especificaciones del programa y procedimientos a seguir se pueden encontrar en Arce et al., (2015). La evaluación del programa se encuentra en la unidad 12, con los materiales bloques I y II, donde se encuentran las fichas para sesiones individuales o de grupo (Arce et al., 2015).

\section{Resultados}

Se realizó un análisis descriptivo para conocer las características sociodemográficas de la muestra, características de las sesiones, asistencia y medir el impacto del tratamiento en los 65 participantes (agresores primarios de género).

Dentro de las características sociodemográficas de la muestra, se reportó un rango mayoritario de edad entre 26 a 35 años (32\%); mayoritariamente su estado civil era el de casados (58\%); el nivel académico básico predominó (45\%), al igual que un nivel económico bajo (49\%) (ver tabla 1). 
Revista lberoamericana

de las Ciencias Sociales y

Humanísticas

ISSN: 2395 - 7972

Tabla 1. Características sociodemográficas de la muestra por edad

\begin{tabular}{|c|c|c|c|c|c|}
\hline \multicolumn{5}{|c|}{$n=65$} & \multicolumn{3}{c|}{ Nivel académico } \\
\hline Rango & Total & $\%$ & Básico & Medio & Superior \\
\hline $15-25$ años & 17 & 26 & 8 & 5 & 4 \\
\hline $26-35$ años & 21 & 32 & 9 & 9 & 3 \\
\hline $36-45$ años & 12 & 18 & 5 & 6 & 1 \\
\hline $46-55$ años & 9 & 14 & 4 & 3 & 2 \\
\hline 56 o más años & 6 & 9 & 3 & 2 & 1 \\
\hline & 65 & 1 & 29 & 25 & 11 \\
\hline & & & $45 \%$ & $38 \%$ & $17 \%$ \\
\hline Estado civil & & Nivel socioeconómico \\
\hline Rango & Casados & Solteros & Bajo & Medio & Alto \\
\hline 15-25 años & 8 & 9 & 10 & 5 & 2 \\
\hline 26-35 años & 10 & 11 & 10 & 6 & 5 \\
\hline $46-45$ años & 8 & 4 & 6 & 5 & 1 \\
\hline 46-55 años & 7 & 2 & 4 & 5 & 0 \\
\hline Totales & 38 & 27 & 32 & 24 & 9 \\
\hline Porcentajes & $58 \%$ & $42 \%$ & $49 \%$ & $37 \%$ & $14 \%$ \\
\hline
\end{tabular}

Fuente: Centros de Atención y Reeducación para Personas que Ejercen Violencia de Género (2019)

Mientras que respecto a las características de las sesiones, la mayoría ingresó al tratamiento con oficio (91\%), provenían del Centro de Atención de Ecatepec (48 \%), el tipo de intervención preponderante fue el mixto $(40 \%)$ y el rango de edad con el promedio más alto de sesiones recibidas fue el de 36 a 45 años ( $83 \%$ ) (ver tabla 2). 
Revista Iberoamericana

de las Ciencias Sociales y

Humanísticas

ISSN: $2395-7972$

Tabla 2. Características de las sesiones de acuerdo con la edad

\begin{tabular}{|c|c|c|c|c|c|}
\hline \multicolumn{6}{|c|}{$n=65$} \\
\hline Edad & \multicolumn{2}{|c|}{ Tipo ingreso } & \multicolumn{3}{|c|}{$\begin{array}{l}\text { Cantidad de pacientes atendidos por centro } \\
\text { de atención }\end{array}$} \\
\hline Rango & Con oficio & Sin oficio & Tlalnepantla & Ecatepec & Atizapán \\
\hline 15-25 años & 15 & 2 & 5 & 8 & 4 \\
\hline 26-35 años & 18 & 3 & 3 & 11 & 7 \\
\hline 36-45 años & 12 & 0 & 3 & 6 & 3 \\
\hline 46-55 años & 9 & 0 & 4 & 5 & 0 \\
\hline 56 o más años & 5 & 1 & 2 & 1 & 3 \\
\hline Totales & 59 & 6 & 17 & 31 & 17 \\
\hline \multirow[t]{2}{*}{ Porcentaje } & $91 \%$ & $9 \%$ & $26 \%$ & $48 \%$ & $26 \%$ \\
\hline & \multicolumn{3}{|c|}{ Tipo de intervención } & $\begin{array}{l}\text { Promedio de } \\
\text { sesiones }\end{array}$ & \\
\hline Rango & Individual & Grupal & Mixta & $\begin{array}{l}\text { recibidas en } \\
\text { el tratamiento }\end{array}$ & $\%$ \\
\hline $15-25$ años & 2 & 4 & 11 & 12 & 50 \\
\hline 26-35 años & 5 & 7 & 9 & 18 & 75 \\
\hline 36-45 años & 6 & 4 & 2 & 20 & 83 \\
\hline 46-55 años & 6 & 1 & 2 & 10 & 42 \\
\hline 56 o más años & 2 & 2 & 2 & 4 & 17 \\
\hline Totales & 21 & 18 & 26 & & \\
\hline Porcentaje & $32 \%$ & $28 \%$ & $40 \%$ & & \\
\hline
\end{tabular}

Fuente: Centros de Atención y Reeducación para Personas que Ejercen Violencia de

Género (2019)

Se realizó el registro del promedio de sesiones que se dio en cada unidad de tratamiento. Aquí se visualizó que el promedio iba acorde al Manual de intervención (Ruiz et al., 2015). También obtuvo el porcentaje de asistencia por unidad de tratamiento para ver el progreso de los pacientes. En este caso se observó el abandono del tratamiento de diez pacientes en la tercera y cuarta unidades por la falta de compromiso e inicio de monitoreo personal y familiar (ver tabla 3). 
Revista Iberoamericana

de las Ciencias Sociales y Humanísticas

ISSN: $2395-7972$

Tabla 3. Comportamiento de asistencia por unidad de tratamiento

\begin{tabular}{|c|c|c|c|c|}
\hline Módulos & Unidades de tratamiento & $\begin{array}{l}\text { Promedio de } \\
\text { sesiones }\end{array}$ & Asistencia & $\%$ \\
\hline $\begin{array}{l}\text { 1) Toma de contacto } \\
\text { e introducción al } \\
\text { programa }\end{array}$ & $\begin{array}{l}\text { 1) Introducción y } \\
\text { diagnóstico }\end{array}$ & 1 & 65 & 100 \\
\hline \multirow[t]{3}{*}{$\begin{array}{l}\text { 2) Responsabilidad } \\
\text { de las conductas } \\
\text { violentas }\end{array}$} & $\begin{array}{l}\text { 2) Responsabilidad de las } \\
\text { conductas violentas: Fase } \\
\text { de sensibilización }\end{array}$ & 2 & 65 & 100 \\
\hline & $\begin{array}{l}\text { 3) Responsabilidad de las } \\
\text { conductas y mecanismos de } \\
\text { defensa }\end{array}$ & 2 & 60 & 92 \\
\hline & $\begin{array}{l}\text { 4) Identificación y } \\
\text { modificación de los estilos } \\
\text { y. monitoreo individual y } \\
\text { familiar }\end{array}$ & 2 & 55 & 85 \\
\hline \multirow[t]{3}{*}{$\begin{array}{l}\text { 3) Concienciación } \\
\text { sobre el ciclo de la } \\
\text { violencia }\end{array}$} & $\begin{array}{l}\text { 5) Formas de violencia de } \\
\text { género y ciclo de la } \\
\text { violencia }\end{array}$ & 3 & 54 & 83 \\
\hline & $\begin{array}{l}\text { 6) Relación entre } \\
\text { situaciones, pensamientos, } \\
\text { emociones y conductas }\end{array}$ & 3 & 52 & 80 \\
\hline & $\begin{array}{l}\text { 7) Identificación y manejo } \\
\text { de situaciones generadoras } \\
\text { de tensión. Reevaluación } \\
\text { psicológica, conductual y } \\
\text { familiar }\end{array}$ & 3 & 46 & 71 \\
\hline $\begin{array}{l}\text { 4) Perspectiva de } \\
\text { género }\end{array}$ & $\begin{array}{l}\text { 8) Confrontación y } \\
\text { reestructuración de } \\
\text { creencias y monitoreo }\end{array}$ & 2 & 44 & 68 \\
\hline $\begin{array}{l}\text { 5) Competencia } \\
\text { personal y social }\end{array}$ & $\begin{array}{l}\text { 9) Emociones y } \\
\text { competencia emocional }\end{array}$ & 2 & 41 & 63 \\
\hline
\end{tabular}


Revista Iberoamericana

de las Ciencias Sociales y

Humanísticas

ISSN: $2395-7972$

\begin{tabular}{|c|c|c|c|c|}
\hline & $\begin{array}{l}\text { 10) Control y regulación } \\
\text { emocional }\end{array}$ & 2 & 39 & 60 \\
\hline & $\begin{array}{l}\text { 11) Autoconcepto y } \\
\text { autoestima }\end{array}$ & 2 & 36 & 55 \\
\hline & $\begin{array}{l}\text { 12) Resolución de } \\
\text { problemas y habilidades de } \\
\text { comunicación }\end{array}$ & 2 & 34 & 52 \\
\hline $\begin{array}{l}\text { 6) Concienciación } \\
\text { sobre el ciclo de la } \\
\text { violencia }\end{array}$ & $\begin{array}{l}\text { 13) Afrontamiento y } \\
\text { prevención de recaídas }\end{array}$ & 2 & 34 & 52 \\
\hline $\begin{array}{l}\text { 7) Cierre y } \\
\text { programación de } \\
\text { seguimiento }\end{array}$ & $\begin{array}{l}\text { 14) Reevaluación } \\
\text { psicológica, conductual y } \\
\text { familiar. Retroalimentación } \\
\text { verbal y escrita }\end{array}$ & 2 & 34 & 52 \\
\hline \multicolumn{2}{|l|}{ Total } & 30 & 34 & 52 \\
\hline
\end{tabular}

Fuente: Centros de Atención y Reeducación para Personas que Ejercen Violencia de Género (2019)

Se realizó el registro de las altas voluntarias o por sistema judicial, ya que existían en las órdenes el tiempo designado para asistir al tratamiento y con alta otorgada por el Centro de Atención con evaluaciones de salida. En la sesión seis se dieron cinco altas requeridas por el Centro de Atención, una con evaluación regular y cuatro con evaluación buena; la restante fue por abandono. De la sesión ocho a la doce, se dieron ocho altas con evaluaciones buenas y tres con evaluaciones regulares, solicitadas por el Centro de Atención y Justicia. Las 34 altas restantes fueron otorgadas en el cierre del tratamiento. En cuanto a las unidades, en “Solución de problemas" se obtuvo un evaluación de 72 \%, en "Habilidades de comunicación con género femenino", $80 \%$ y en "Conductas violentas contra género femenino", $70 \%$ (ver tabla 4). 
Revista lberoamericana

de las Ciencias Sociales y

Humanísticas

ISSN: 2395 - 7972

Tabla 4. Impacto del tratamiento

\begin{tabular}{|c|c|c|c|c|c|}
\hline \multirow[b]{2}{*}{ 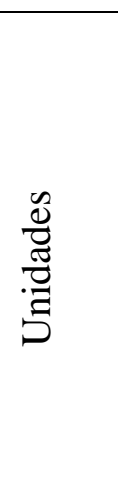 } & \multirow[t]{2}{*}{ Altas: voluntaria/sistemática } & \multirow[t]{2}{*}{$\%$} & \multicolumn{3}{|c|}{ Evaluaciones de salida } \\
\hline & & & $\begin{array}{c}\text { Soluciones } \\
\text { de } \\
\text { problemas } \\
\text { cotidianos }\end{array}$ & $\begin{array}{l}\text { Habilidades } \\
\text { de } \\
\text { comunicación } \\
\text { género } \\
\text { femenino }\end{array}$ & $\begin{array}{l}\text { Comportamiento } \\
\text { violento contra el } \\
\text { género femenino }\end{array}$ \\
\hline 1 & 0 & 0 & - & - & - \\
\hline 2 & 0 & 0 & - & - & - \\
\hline 3 & 5 & 8 & 5 malas & 5 malas & 6 malas \\
\hline 4 & 5 & 8 & $\begin{array}{c}3 \text { malas y } \\
2 \\
\text { regulares }\end{array}$ & $\begin{array}{c}2 \text { malas y } 3 \\
\text { regulares }\end{array}$ & $\begin{array}{c}3 \text { malas y } 3 \\
\text { regulares, } 10 \\
\text { regulares }\end{array}$ \\
\hline 5 & 1 & 2 & $1 \mathrm{mala}$ & $1 \mathrm{mala}$ & 2 mala \\
\hline 6 & 2 & 3 & $\begin{array}{c}1 \text { malas y } \\
1 \\
\text { regulares }\end{array}$ & 2 regulares & 2 regulares \\
\hline 7 & 6 & 9 & $\begin{array}{c}1 \text { mala, } 1 \\
\text { regular y } 4 \\
\text { buenas }\end{array}$ & $\begin{array}{c}2 \text { regular y } 4 \\
\text { buenas }\end{array}$ & $\begin{array}{c}3 \text { regular y } 4 \\
\text { buenas }\end{array}$ \\
\hline 8 & 2 & 3 & $\begin{array}{c}1 \text { regular y } \\
1 \text { buena }\end{array}$ & 2 buena & 2 buena \\
\hline 9 & 3 & 5 & $\begin{array}{c}1 \text { regular y } \\
2 \text { buena }\end{array}$ & $\begin{array}{c}1 \text { regular y } 2 \\
\text { buena }\end{array}$ & $\begin{array}{c}2 \text { regular y } 1 \\
\text { buena }\end{array}$ \\
\hline 10 & 2 & 3 & $\begin{array}{c}1 \text { regular y } \\
1 \text { buena }\end{array}$ & 2 buena & $\begin{array}{c}1 \text { regular y } 1 \\
\text { buena }\end{array}$ \\
\hline 11 & 3 & 5 & 3 buenas & 3 buenas & 2 buenas \\
\hline 12 & 2 & 3 & 2 buenas & 2 buenas & 2 buenas \\
\hline Cierre & 34 & 52 & 34 buenas & 34 buenas & 34 buenas \\
\hline Total & 65 & 100 & & & \\
\hline
\end{tabular}


Revista Iberoamericana

de las Ciencias Sociales y

Humanísticas

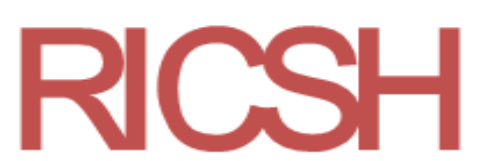

ISSN: 2395 - 7972

\begin{tabular}{|l|c|c|l|}
\hline Porcentaje de impacto & $\begin{array}{c}11 \text { malas }(17 \%), \\
7 \text { regulares }\end{array}$ & $\begin{array}{c}5 \text { malas } \\
(8 \%), 8\end{array}$ & 11 malas (17\%), \\
& $(11 \%)$ y 47 & regulares \\
buenas $(72 \%)$ & $(12 \%)$ y 52 & buenas (70 \%) \\
& & buenas & \\
& & $(80 \%)$ & \\
\hline
\end{tabular}

Fuente: Centros de Atención y Reeducación para Personas que Ejercen Violencia de

Género (2019)

\section{Discusión}

La investigación prueba un tratamiento para el agresor primario de género para disminuir la violencia de género. Sin embargo, es importante señalar que aquí, por ser un problema multifactorial, solo se está atendiendo una parte. Como lo menciona Bejarano (2014), la violencia hacia las mujeres debe ser atendida de manera integral. Aun con todo, la parte atendida en este estudio es muy importante, porque brinda tratamiento a las ideas y conductas del victimario, generador de la violencia de género y feminicidio.

Existen otros esfuerzos para disminuir el problema de la violencia de género dirigidas a las víctimas. Se trata de acciones que pueden ser complementarias y resolver en parte este grave problema, que tiene muchas aristas, como también lo dejan claro Russell y Radford (2006). Por ser un problema de índole social y cultural, se requiere de la intervención de los derechos humanos y el poder judicial. Y sobre todo de acciones conjuntas que cambien la forma de pensar y actuar de la víctima como del victimario (Castañeda, et al., 2013).

Bien es cierto que existe un número considerable de programas dirigidos a las víctimas, pero también hay que atender la causa del problema, "el pensamiento de superioridad del victimario" (Arce et al., 2014). Cabe recordar que el victimario ejerce un rol de poder porque socialmente es fomentado, reforzado y no castigado. El reaprendizaje a través de un tratamiento brinda al victimario la justicia terapéutica para reivindicarse ante la sociedad y cambiar su forma de pensar y actuar. El victimario requiere modificar sus creencias o el núcleo de su actuación. Si se controla a los generadores de la violencia de género, se podrá incidir en el feminicidio (Bejarano, 2014). Esta es una forma de control o modificación de pensamiento e impulsos. 


\section{Revista Iberoamericana \\ de las Ciencias Sociales y Humanísticas}

ISSN: 2395 - 7972

Se ha demostrado que los agresores no son enfermos mentales que actúan sin responsabilidad del acto, sino son sujetos que tienen problemas conductuales, generados por pensamientos aprendidos de minusvalía del género femenino (Lila et al, 2012). La aplicación de un tratamiento programado, integral, y flexible es una buena alternativa para disminuir la agresión de género (Arce et al., 2015).

Es importante señalar que el tratamiento se ajustó tanto a las evaluaciones psicológicas y comportamentales como a las situaciones de casos. Respecto a estas últimas, se trata de las sentencias específicas en tiempo para el tratamiento, los procesos legales de ejecución o la falta de interés de los participantes por cohesión social, factores que funcionan como flagelos para el victimario porque retrasan la recuperación (Castañeda et al., 2013).

\section{Conclusiones}

Originalmente, el tratamiento está diseñado para 52 sesiones y aquí se ajustó a 30 sesiones por requerimientos judiciales y personales. Así, se observó un impacto en la solución de problemas cotidianos (72\%), mejora de habilidades de comunicación con el género femenino $(80 \%)$ y la concientización y reprobación de conductas violentas en contra el género femenino $(70 \%)$, factores que sin duda contribuyen a disminuir el riesgo de feminicidio. Es necesario continuar con el registro y seguimiento, apoyando en el afrontamiento de su realidad y para la prevención de recaídas. La recuperación total e integración social del victimario es esencial para la familia, "núcleo de la sociedad", donde la equidad de género es vital para que la sociedad funcione de forma integral y equilibrada.

\section{Futuras líneas de investigación}

De acuerdo con la investigación realizada, podemos decir que los hallazgos del trato psicológico a los victimarios son muy interesantes, porque ellos son personas que sufren por los problemas de la vida cotidiana y que sus reacciones son un reflejo de lo aprendido, y cuando se les atiende, ellos pueden visualizar sus errores de conducta y el impacto que tiene tanto en su vida personal como familiar, entonces los victimarios empiezan a realizar el cambio. Las nuevas investigaciones serán implementar otros tipos de tratamientos para poblaciones tipificadas como agresores primarios de género y así poder comparar el impacto de estos y tener diferentes alternativas que puedan ayudar a disminuir la violencia de género. 
Revista Iberoamericana

de las Ciencias Sociales y

Humanísticas

ISSN: $2395-7972$

\section{Referencias}

Arce, R. y Fariña, F. (2005). Entrevista semiestructurada para maltratadores de género. (Manuscrito no publicado). Universidad de Santiago de Compostela, Santiago de Compostela.

Arce, R. y Fariña, F. (2010). Diseño e implementación del programa Galicia de Reeducación de Maltratadores: una respuesta psicosocial a una necesidad social y penitenciaria. Intervención Psicosocial, 19(2), 153-166. Recuperado de http://scielo.isciii.es/scielo.php?script=sci_arttext\&pid=S113205592010000200007.

Arce, R., Fariña, F. y Novo, M. (2014). Competencia cognitiva en penados primarios y reincidentes: implicaciones para la reeducación. Anales de Psicología, 30(1), 259266. Recuperado de http://scielo.isciii.es/scielo.php?script=sci_arttext\&pid=S021297282014000100027.

Arce, R., Fariña, F., Vázquez, M. J., Novo, M. y Seijo, D. (2015). Programa Galicia de Reeducación de Agresores de Género. Manual de intervención. Santiago de Compostela, España: Andavira Editora.

Arteaga, N. y Valdés, J. (2010). Contextos socioculturales de los feminicidios en el Estado de México: nuevas subjetividades femeninas. Revista Mexicana de Sociología, 72(1), 5-35.

Bejarano, C. M. (2014). El feminicidio es sólo la punta del iceberg. Religión y Sociedad, (4), 13-44. Recuperado de http://www.redalyc.org/articulo.oa?id=10230108002.

Butcher, J. N., Graham, J. R., Ben-Porath, Y. S., Tellegen, A. y Grant, W. (1995). Inventario multifacético de la personalidad de Minnesota. Manual de aplicación, calificación e interpretación (2. ${ }^{a}$ ed.). Manual Moderno.

Castañeda, M. P., Ravelo, P. y Pérez, T. (2013). Feminicidio y violencia de género en México: omisiones del Estado y exigencia civil. Revista de Ciencias Sociales y Humanidades, 74(34), 11-39. Recuperado de https://www.redalyc.org/html/393/39348328002/.

Comisión de Derechos Humanos del Distrito Federal. (2011). Editorial. dfensor,(3), 3.

Congreso de la Unión. (14 de junio de 2012). Decreto por el que se reforman y adicionan diversas disposiciones del Código Penal Federal, de la Ley General de Acceso de las Mujeres a una Vida Libre de Violencia, de la Ley Orgánica de la Administración 
Revista Iberoamericana

de las Ciencias Sociales y

Humanísticas

ISSN: $2395-7972$

Pública Federal y de la Ley Orgánica de la Procuraduría General de la República. Diario Oficial de la Federación. Recuperado de http://www.dof.gob.mx/nota_detalle.php?codigo=5253274\&fecha=14/06/2012.

García, A. K. (13 de febrero de 2021). Sólo en los primeros seis meses del 2020 fueron asesinadas 1,844 mujeres en México: Inegi. El economista. Recuperado de https://www.eleconomista.com.mx/politica/Solo-en-los-primeros-seis-meses-del2020-fueron-asesinadas-1844-mujeres-en-Mexico-Inegi-20210213-0002.html.

Gobierno de México. (2021). Incidencia delictiva nacional. Presuntos delitos registrados en marzo: $183111 . \quad$ Recuperado de https://drive.google.com/file/d/19DZITq0EQCoBzLlimnjXm3AYQNplWyWC/vie w.

Guerra, F. (2018). \#NiUnaMás. El feminicidio en México: tema urgente en la agenda nacional. México: Aguilar.

Instituto Nacional de Estadística y Geografía [Inegi]. (18 de agosto de 2017). Encuesta Nacional sobre la Dinámica de las Relaciones en los Hogares (Endireh) 2016. Principales resultados. México: Instituto Nacional de Estadística y Geografía. Recuperado de https://www.inegi.org.mx/contenidos/programas/endireh/2016/doc/endireh2016_pre sentacion_ejecutiva.pdf.

Instituto Nacional de Estadística y Geografía [Inegi]. (22 de noviembre de 2018). Estadísticas a propósito del Día Internacional de la Eliminación de la Violencia contra la Mujer (25 de noviembre) / Datos nacionales. Comunicado de prensa, (588/18). Recuperado de https://www.inegi.org.mx/contenidos/saladeprensa/aproposito/2018/violencia2018_ Nal.pdf.

Jiménez, N. P. (2011). Femicidio/feminicidio: una salida emergente de las mujeres frente a la violencia ejercida en contra de ellas. Revista Logos, Ciencia \& Tecnología, 3(1) 127-148.

LIII Legislatura del Estado de México. (20 de marzo de 2020). Código Penal del Estado de México. Gaceta del Gobierno. Recuperado de https://legislacion.edomex.gob.mx/sites/legislacion.edomex.gob.mx/files/files/pdf/c od/vig/codvig006.pdf. 
Revista Iberoamericana

de las Ciencias Sociales y

Humanísticas

ISSN: 2395 - 7972

Lila, M., Gracia, E. y Herrero, J. (2012). Asunción de responsabilidad en hombres maltratadores: influencia de la autoestima, la personalidad narcisista y la personalidad antisocial. Revista Latinoamericana de Psicología, 44(2), 99-108.

López, M. M. (2010). La violencia de género en el territorio latinoamericano, a través de la ocurrencia creciente de los feminicidios en la región. Revista Latinoamericana de Geografía de Género, Ponta Grossa, 1(1), 78-88. Recuperado de http://alianzaintercambios.net/files/doc/1269315363_La\%20violencia\%20de\%20G $\% \mathrm{C} 3 \%$ A9nero.pdf.

Monárrez, J. (2010). Las diversas representaciones del feminicidio y los asesinatos de mujeres en Ciudad Juárez, 1993- 2005. En Monárrez, J., Cervera, L., Fuentes, C. y Rubio, R. (coords.), Violencia contra las mujeres e inseguridad ciudadana en Ciudad Juárez. México: El Colegio de la Frontera Norte/Miguel Ángel Porrúa.

Moscovici, S. (2002). Psicología social II. Pensamiento y vida social: psicología social y problemas sociales. Barcelona, España: Paidós.

Pérez, M., Giménez, F. y Espinosa, M. (2012). Evaluación del programa "Violencia de Género: programa de intervención para agresores”, en medidas alternativas. Madrid, España: Secretaría General de Instituciones Penitenciarias, Ministerio del Interior y el Instituto de Ciencias Forenses y de la Seguridad de la Universidad Autónoma de Madrid. Recuperado de http://www.institucionpenitenciaria.es/web/export/sites/default/datos/descargables/d es cargas/VDG_EVALUACION_AUTONOMA_NIPO.pdf.

Organización de las Naciones Unidas [ONU]. (2007). Informe del Comité para la Eliminación de la Discriminación contra la Mujer. Nueva York, Estados Unidos: Organización de las Naciones Unidas. Recuperado de https://digitallibrary.un.org/record/760503/files/A_68_44-ES.pdf.

Organización Mundial de la Salud [OMS]. (2011). La prueba de detección de consumo de alcohol, tabaco y sustancias Manual para uso en la atención primaria. Washington, Estados Unidos: Organización Mundial de la Salud.

Organización Mundial de la Salud [OMS]-Organización Panamericana de la Salud [OPS]. (22 de noviembre de 2017). Respondiendo a la violencia contra las mujeres: qué deberían hacer los servicios de salud. Recuperado de https://www3.paho.org/hq/index.php?option=com_content\&view=article\&id=1394 
Revista Iberoamericana

de las Ciencias Sociales y

Humanísticas

ISSN: $2395-7972$

5:responding-to-violence-against-women-what-health-services-can-

do\&Itemid=135\&lang=es.

Raven, J. C. (2015). Test de matrices progresivas. Escala avanzada (5. a ed.). Barcelona, España: Paidós.

Romero, T. I. (2014). Sociología y política del feminicidio; algunas claves interpretativas a partir de caso mexicano. Sociedad y Estado, 29(2) 375-391. Recuperado de http://www.scielo.br/scielo.php?script=sci_arttext\&pid=S010269922014000200004.

Russell, D. E. y Radford, J. (2006). Feminicidio. La política del asesinato de las mujeres. Ciudad de México, México: Universidad Nacional Autónoma de México.

Ruiz, S., Negredo, L., Ruiz, A., García, C., Herrero, O., Yela, M. y Pérez, M. (2010). Violencia de género. Programa de intervención para agresores (PRIA). Madrid, España: Ministerio del Interior. Secretaría General Técnica.

Salvatierra, K. S. (2007). Reseña de "Feminicidio. La política del asesinato de las mujeres" de Diana E. Russell y Hill Radford (eds.). Revista Mexicana de Ciencias Políticas y Sociales, $\quad 49(200), \quad$ 169-171. Recuperado de http://www.redalyc.org/pdf/421/42120010.pdf.

Secretaría de Gobernación. (1 de julio de 2020). Código Penal Federal. Diario Oficial de la Federación. Recuperado de http://www.cegaipslp.org.mx/HV2020Dos.nsf/nombre_de_la_vista/92DDC5FFBDF 2A334862585CE005EE52B/\$File/Codigo+Penal+federal.pdf.

Sociedad Mexicana de Psicología. (2010). Código ético del psicólogo. México: Trillas. 